\title{
GENERALIZED POWER SERIES FIELDS
}

\author{
BY \\ PHILIP W. CARRUTH
}

1. Introduction. A field with a valuation is said to be maximal if every extension of the field must enlarge either the value group or the residue class field. Kaplansky [1 and 2] $\left.{ }^{(}\right)$has shown that if the characteristic of a maximal field is the same as that of its residue class field, under certain conditions the maximal field is analytically isomorphic to a power series field.

It is the purpose of this paper to state criteria not dependent on the characteristics of a maximal field and its residue class field for a maximal field to be analytically isomorphic to a power series field.

In order to accomplish this purpose, a generalized power series field is constructed in \$2. In the process of showing that this field is maximal in its natural valuation, a result is obtained concerning the cardinal number of a maximal field. In $\$ 3$, our main result is obtained.

2. Generalized power series fields. Throughout this paper, $K$ will denote a field with a valuation $V$ on an ordered abelian group $\Gamma$, and $\Omega$ will denote the corresponding residue class field.

Let $M$ be a system of representatives in $K$ of the residue classes in $\Omega$. In particular, let 0 and 1 in $K$ be the representatives of 0 and 1 respectively in $\Omega$. If $a$ is in $K$ and $V(a) \geqq 0$, then let $a^{\prime}$ be the element in $M$ that belongs to the same residue class as $a$, and let $B[a]=a-a^{\prime}$.

Let $G$ be a system of representatives in $K$ of the values in $\Gamma$. In particular, let 1 in $K$ be the representative of 0 in $\Gamma$. If $\alpha$ is any element in $\Gamma$, let $t^{\alpha}$ be the element in $G$ whose value is $\alpha$. For $x \neq 0$ in $K$ and $\alpha$ in $\Gamma$, let $c(x, \alpha)$ $=x t^{\alpha} / t^{V(x)+\alpha}$. Let $c(0, \alpha)=1$ in $K$. It is noted that $V[c(x, \alpha)]=0$.

Let $C_{1}$ be the set of all products of the form $\prod_{i=1}^{n} c\left(x_{i}, \alpha_{i}\right)$, for all $x_{i}$ in $G, \alpha_{i}$ in $\Gamma$, and every positive integer $n$. Let $L_{1}$ be the set of all elements of the form $B$ [ $\left.\sum_{i=1}^{n} a_{i} b_{i} d_{i}\right]$, for all $a_{i}, b_{i}$, in $M, d_{i}$ in $C_{1}$, and every positive integer $n$.

Assume that for every positive integer $i<k, C_{i}$ and $L_{i}$ have been defined. Then $C_{k}$ is the set of all products of the form $\prod_{i=1}^{n} c_{i} \prod_{i=1}^{m} c\left(x_{i}, 0\right)$, for all $c_{i}$ in $C_{k-1}$, all $x_{i}$ in $L_{k-1}$, and every pair of positive integers $n$ and $m . L_{k}$ is the set of all elements of the form $B\left[\sum_{i=1}^{n} a_{i} b_{i} d_{i}\right]$, for all $a_{i}, b_{i}$ in $M$, all $d_{i}$ in $C_{k}$, and every positive integer $n$.

Let $C$ be the join of all the $C_{i}$, and $L$ the join of all the $L_{i}$. It is seen immediately that:

1. $B$ [ $\left.\sum_{i=1}^{n} a_{i} b_{i} d_{i}\right]$ is in $L$ for all $a_{i}, b_{i}$ in $M$, all $d_{i}$ in $C$, and for every positive integer $n$.

Presented to the Society, April 25, 1947; received by the editors April 16, 1947.

(1) Numbers in brackets refer to the bibliography at the end of the paper. 
2. If $c$ and $d$ are in $C$, then $c d$ is in $C$.

3. If $x$ is in $L$ or in $G$, and $\alpha$ is in $\Gamma$, then $c(x, \alpha)$ is in $C$.

Now let $\theta$ be the set of all finite sums of the values of the elements in $L$ and 0 . We impose the following condition:

CondiTion 2.1. There are at most a finite number of elements in $\theta$ that are less than any given element in $\Gamma$.

This condition implies that the set $\theta$ is well-ordered according to the ordering of $\Gamma$ and that its order type is not greater than $\omega$. $(\theta$ might consist solely of integral multiples of a single element in $\Gamma$, although this would not be necessarily so. For example, if $\Gamma$ were the additive group of rational numbers, $\theta$ might consist of all finite sums of the elements $2,2+1 / 2$, $3+1 / 3, \cdots, n+1 / n, \cdots$.

If $\phi$ is any subset of $\Gamma$ that is well-ordered according to the ordering in $\Gamma$, if $a_{\alpha}$, for every $\alpha$ in $\phi$, is an element in $M$, then we term $\sum_{\alpha} a_{\alpha} t^{\alpha}, \alpha \in \phi$, a formal power series (over $M, G$ ).

The power series $\sum_{\alpha} a_{\alpha} t^{\alpha}, \alpha \in \phi$, and $\sum_{\beta} b_{\beta} t^{\beta}, \beta \in \psi$, are equal if, and only if :

1. " $\alpha \in \phi, \alpha \notin \psi$ " implies that $a_{\alpha}=0$.

2. " $\beta \notin \phi, \beta \in \psi$ " implies that $b_{\beta}=0$.

3. " $\alpha \in$ both $\phi$ and $\psi$ " implies that $a_{\alpha}=b_{\alpha}$.

It can be verified that this equality relation is symmetric, reflexive, and transitive.

Thus two power series are equal if, and only if, each may be obtained from the other by the insertion and/or deletion of terms $a_{\alpha} t^{\alpha}$, where $a_{\alpha}=0$.

We denote by $M\left(t^{\mathrm{r}}\right)$ the set of all formal power series (over $M, G$ ), subject to the above definition of equality.

2.2. Addition in $M\left(t^{\Gamma}\right)$. Let $A=\sum_{\alpha} a_{\alpha} t^{\alpha}, \alpha \in \phi$, and $B=\sum_{\beta} b_{\beta} t^{\beta}, \beta \in \psi$, be any two elements in $M\left(t^{\mathrm{r}}\right)$.

$A+B=D=\sum_{\delta} d_{\delta} t^{\delta}, \delta \in \Delta$, where $\Delta$ is the set of all elements of the form $\alpha+\gamma, \beta+\gamma$, for all $\alpha$ in $\phi$, all $\beta$ in $\psi$, and all $\gamma$ in $\theta$.

If there is no element $\alpha$ in $\phi$ equal to a given element $\delta$ in $\Delta$, let $a_{8}=0$. If there is no element $\beta$ in $\psi$ equal to a given element $\delta$ in $\Delta$, let $b_{\delta}=0$.

Let $\epsilon$ be the smallest element in $\Delta$. Then $d_{\epsilon}=\left(a_{\epsilon}+b_{\epsilon}\right)^{\prime}$, and $B[\epsilon]=B\left[a_{\epsilon}+b_{\epsilon}\right]$. Assume that the elements $d_{\rho}$ and $B[\rho]$ have been defined for all $\rho$ in $\Delta$ that are less than $\delta$ in $\Delta$, and that for each of these $\rho, B[\rho]$ is in $L$. For each of these $\rho$, let $c_{\rho}=c(B[\rho], \rho)$.

Then $d_{\delta}=\left(a_{\delta}+b_{\delta}+\sum_{\rho} c_{\rho}\right)^{\prime}$, where $\rho$ runs over all elements in $\Delta$ having the property $V(B[\rho])+\rho=\delta$. Since the elements in $\Delta$ and in $\theta$ are wellordered, $\sum c_{\rho}$ is a finite summation.

$B[\delta]=B\left[a_{\delta}+b_{\delta}+\sum c_{\rho}\right]$ is in $L$, since each $c_{\rho}$ is in $C . B[\delta]$ is the surplus of $d_{\delta}$.

2.3 Multiplication in $M\left(t^{\mathrm{\Gamma}}\right)$. Let $A$ and $B$ be defined as in 2.2.

$A B=D=\sum_{\delta} d_{\delta} t^{\delta}, \delta \in \Delta$, where $\Delta$ is the set of all elements of the form $\alpha+\beta+\gamma$, for all $\alpha$ in $\phi$, all $\beta$ in $\psi$, and all $\gamma$ in $\theta$. 
Let $\sigma$ be the smallest element in $\phi$, and let $\tau$ be the smallest element in $\psi$. Then $\sigma+\tau=\epsilon$ is the smallest element in $\Delta$.

$$
d_{\epsilon}=\left[a_{\sigma} b_{\tau} c\left(t^{\sigma}, \tau\right)\right]^{\prime} \text { and } B[\epsilon]=B\left[a_{\sigma} b_{\tau} c\left(t^{\sigma}, \tau\right)\right] .
$$

Assume the elements $d_{\rho}$ and $B[\rho]$ have been defined for all $\rho$ in $\Delta$ that are less than $\delta$ in $\Delta$, and that for each of these $\rho, B[\rho]$ is in $L$. For each of these $\rho$, let $c_{\rho}=c(B[\rho], \rho)$.

Then $d_{\delta}=\left(\sum_{\lambda, \mu} a_{\lambda} b_{\mu} c\left(t^{\lambda}, \mu\right)+\sum_{\rho} c_{\rho}\right)^{\prime}$, where $\lambda$ and $\mu$ run over all elements in $\phi$ and $\psi$ respectively such that $\lambda+\mu=\delta$, and where $\rho$ runs over all elements in $\Delta$ that have the property $\rho+V(B[\rho])=\delta . B[\delta]=B\left[\sum a_{\lambda} b_{\mu} c\left(t^{\lambda}, \mu\right)+\sum c_{\rho}\right]$ is in $L . B[\delta]$ is the surplus of $d_{\delta}$.

THEOREM 2.4. With addition and multiplication defined as in 2.2 and 2.3 respectively, $M\left(t^{\mathbf{\Gamma}}\right)$ is a field.

Proof. Addition and multiplication in $M\left(t^{\mathrm{r}}\right)$ are both obviously commutative. We now show that addition is unique. Consider the power series $\sum a_{\alpha} t^{\alpha}$ $=\sum w_{\mu} t^{\mu}, \alpha \in \phi$ and $\mu \in \pi$, and the series $\sum b_{\beta} t^{\beta}=\sum u_{\tau} t^{\tau}, \beta \in \psi$ and $\tau \in \Omega$.

We first assume that $\pi$ is contained in $\phi$ and that $\psi$ is equal to $\Omega$. Then $\sum a_{\alpha} t^{\alpha}+\sum b_{\beta} t^{\beta}=\sum d_{\delta} t^{\delta}, \delta \in \Delta$, and $\sum w_{\mu} t^{\mu}+\sum b_{\beta} t^{\beta}=\sum m_{\lambda} t^{\lambda}, \lambda \in \Lambda$.

It is seen that $\Lambda$ is contained in $\Delta$. Let $\epsilon$ be the smallest element in $\Delta$. Suppose $\epsilon$ is not in $\Lambda$. Then $\epsilon$ is in $\phi$, but in neither $\pi$ nor $\psi$. Hence $a_{\epsilon}=0, d_{\epsilon}=0$.

Now suppose that $\epsilon$ is in $\Lambda$. Then $\epsilon$ is the smallest element in $\Lambda$, and $m_{\epsilon}=\left(w_{\epsilon}+b_{\epsilon}\right)^{\prime} . d_{\epsilon}=\left(a_{\epsilon}+b_{\epsilon}\right)^{\prime}$. Since $a_{\epsilon}=w_{\epsilon}$, it follows that $m_{\epsilon}=d_{\epsilon}$.

For every $\rho$ in $\Delta$ less than $\delta$ in $\Delta$, assume that $d_{\rho}=0$ for $\rho$ not in $\Lambda$, and $d_{\rho}=m_{\rho}$ for $\rho$ in $\Lambda$, and that the surplus of $d_{\rho}$ equals 0 for $\rho$ not in $\Lambda$, and the surplus of $d_{\rho}$ equals the surplus of $m_{\rho}$ for $\rho$ in $\Lambda$.

Let $d_{\delta}=\left(a_{\delta}+b_{\delta}+\sum c_{\rho}\right)^{\prime}$, as in 2.2. Suppose first that $\delta$ is not in $\Lambda$. Then $\delta$ is in neither $\pi$ nor $\psi$, and so $a_{\delta}=b_{\delta}=0$. Likewise by our induction hypothesis, there can be no element $\rho$ in $\Delta$ such that $V(B[\rho])+\rho=\delta$. For if there were, $B$ [o] would not equal 0 , and so $\rho$ and hence also $\delta$ would be in $\Lambda$. Therefore $d_{\delta}=\left(a_{\delta}+b_{\delta}\right)^{\prime}=0$.

Suppose now that $\delta$ is in $\Lambda . m_{\delta}=\left(w_{\delta}+b_{\delta}+\sum c_{\rho}\right)^{\prime}$, by our induction hypothesis. But $w_{\delta}=a_{\delta}$. Hence $m_{\delta}=d_{\delta}$.

It can be verified easily now that in every case addition in $M\left(t^{\mathbf{r}}\right)$ is unique. In a similar manner it can be shown that multiplication in $M\left(t^{\mathrm{r}}\right)$ is unique.

Now we shall show that addition and multiplication are associative and that the distributive law holds.

Let $A=\sum a_{\alpha} t^{\alpha}, B=\sum b_{\alpha} t^{\alpha}$ and $C=\sum c_{\alpha} t^{\alpha}, \alpha \in \phi$, be any three elements in $M\left(t^{\mathrm{r}}\right)$.

We may assume without loss of generality that the element 0 is in $\phi$. Let $\Delta^{\prime}$ be the set of all elements of the form $\alpha+\gamma$, for all $\alpha$ in $\phi$ and all $\gamma$ in $\theta$. Let $\Delta^{\prime \prime}$ be the set of all elements of the form, $\alpha+\alpha^{\prime}+\gamma$, for all $\alpha, \alpha^{\prime}$ in $\phi$ and 
all $\gamma$ in $\theta$. Let $\Delta$ be the set of all elements of the form $\alpha+\alpha^{\prime}+\alpha^{\prime \prime}+\gamma$, for all $\alpha, \alpha^{\prime}, \alpha^{\prime \prime}$ in $\phi$ and all $\gamma$ in $\theta$. Then $\Delta^{\prime}$ contains $\phi, \Delta^{\prime \prime}$ contains $\Delta^{\prime}$, and $\Delta$ contains $\Delta^{\prime \prime}$.

$$
\begin{aligned}
& (A+B)+C=\sum r_{\delta} t^{\delta}, \quad A+(B+C)=\sum p_{\delta} t^{\delta}, \\
& (A B) C=\sum d_{\delta} t^{\delta}, \quad A(B C)=\sum s_{\delta} t^{\delta}, \\
& (A+B) C=\sum n_{\delta} t^{\delta}, \quad A C+B C=\sum y_{\delta} t^{\delta}, \quad \delta \in \Delta .
\end{aligned}
$$

Let $\sigma$ be the smallest element in $\phi$. We may assume that $a_{\sigma}=b_{\sigma}=c_{\sigma}=0$. Let $\epsilon$ be the smallest element in $\Delta$. Clearly, then, $r_{\epsilon}=p_{\epsilon}, d_{\epsilon}=s_{e}, n_{\epsilon}=y_{e}$. Assume that for all $\rho$ in $\Delta$ less than $\delta$ in $\Delta, r_{\rho}=p_{\rho}, d_{\rho}=s_{\rho}$, and $n_{\rho}=y_{\rho}$.

Let $A^{\prime}, B^{\prime}, C^{\prime}$ be the sets of all elements $a_{\alpha}, b_{\alpha}, c_{\alpha}$ respectively for all $\alpha$ in $\phi$.

LEMMA 2.5. There is a subset $\phi_{\delta}(A, B)$ of $\phi$ that contains all but a finite number of the elements in $\phi$, and such that the coefficients of $t^{8}$ in $A+B$ and $A B$ and the surplus of each of these coefficients remains unchanged if each element in $A^{\prime}$ and each element in $B^{\prime}$ with a subscript in $\phi_{\delta}(A, B)$ is replaced by an element in $M$.

Proof. $\phi_{\epsilon}(A, B)$ consists of every element in $\phi$ except $\sigma$. Assume that for every $\rho$ in $\Delta$ less than $\delta$ in $\Delta, \phi_{\rho}(A, B)$ has been determined.

Let $Q_{\delta}$ be the finite set of elements in $\phi$ of the form $\delta-\tau$, where $\tau$ is any element in $\phi$. It is seen that $Q_{\delta}$ contains $\delta$ if $\phi$ contains $\delta$.

Then $\phi_{\delta}(A, B)=\prod_{\rho} \phi_{\rho}(A, B)-Q_{\delta}$, where $\prod$ denotes set intersection, and where $\rho$ runs over the finite set of elements in $\Delta$ of the form $\delta-\gamma$, where $\gamma$ is any element in $\theta$.

It is seen that for each of these $\rho, \phi_{\rho}(A, B)$ contains $\phi_{\delta}(A, B)$. Hence for each of these $\rho$, the surplus of the coefficients of $t^{\rho}$ in $A+B$ and $A B$ would not be changed by the replacement of each element in $A^{\prime}$ and each element in $B^{\prime}$ with a subscript in $\phi_{\delta}(A, B)$ by an element in $M$. Hence the coefficients of $t^{8}$ in $A+B$ and $A B$ and the surplus of each would not be changed. It is seen that $\phi_{8}(A, B)$ contains all but a finite number of the elements in $\phi$.

We use the notation of 2.5 and let $\psi_{1}=\Delta_{\delta}^{\prime}(A+B, C) \Delta_{\delta}^{\prime}(A, B+C)$, and let $\psi_{2}=\Delta_{\delta}^{\prime \prime}(A B, C) \Delta_{\delta}^{\prime \prime}(A, B C) \Delta_{\delta}^{\prime \prime}(A C, B C)$.

Let $\rho(1), \cdots, \rho(k)$ be the finite set of elements in $\Delta^{\prime}$ that are not in $\psi_{1}$. Let $\rho(k+1), \cdots, \rho(n)$ be the finite set of elements in $\Delta^{\prime \prime}$ that are not in $\psi_{2}$.

Let $\Omega=\prod_{i=1}^{n} \phi_{\rho(i)}(A, B) \prod_{i=1}^{n} \phi_{\rho(i)}(B, C) \prod_{i=1}^{n} \phi_{\rho(i)}(A, C)$. It is noted that no $\rho(i)$ is in $\Omega$ and so that every element in $\Omega$ is in both $\psi_{1}$ and $\psi_{2}$.

$\Omega$ contains all but a finite number of the elements in $\phi$. Let $D^{\prime}$ be the set of all elements in the sets $A^{\prime}, B^{\prime}, C^{\prime}$ that have subscripts in $\Omega$. $D^{\prime}$ contains all but a finite number of the elements in the sets $A^{\prime}, B^{\prime}$, and $C^{\prime}$.

We replace each element in $D^{\prime}$ by 0 . By 2.5 , since each element in $\Omega$ also appears in each $\phi_{\rho(i)}(A, B)$, the coefficients of $t^{\rho(i)}$ in $A+B$ and $A B$ are left unchanged. Every element in $\Delta^{\prime}$ not a $\rho(i)$ is in $\psi_{1}$ and so is in $\Delta_{\delta}^{\prime}(A+B, C)$. Therefore $r_{\delta}$ is left unchanged. Every element in $\Delta^{\prime \prime}$ not a $\rho(i)$ is in $\psi_{2}$ and so 
in $\Delta_{\delta}^{\prime \prime}(A B, C)$. Hence $d_{\delta}$ is left unchanged. Similarly it is seen that $p_{\delta}$ and $s_{\delta}$ are left unchanged.

Every element in $\Delta^{\prime}$ not a $\rho(i)$ is in $\Delta_{\delta}^{\prime}(A+B, C)$. Hence $n_{\delta}$ is left unchanged. Since every element in $\Omega$ also appears in each $\phi_{\rho(i)}(A, C)$ and in each $\phi_{\rho(i)}(B, C)$, the coefficients of each $t^{\rho(i)}$ in $A C$ and $B C$ are left unchanged. Every element in $\Delta^{\prime \prime}$ not a $\rho(i)$ is in $\Delta_{\delta}^{\prime \prime}(A C, B C)$, and so $y_{\delta}$ is not changed.

By replacing each element in $D^{\prime}$ by $0, A, B$, and $C$ can be reduced to the finite summations, $\bar{A}=\sum a_{\beta} t^{\beta}, \bar{B}=\sum b_{\beta} t^{\beta}$, and $\bar{C}=\sum c_{\beta} t^{\beta}$ respectively. $\bar{A}, \bar{B}$, and $\bar{C}$ may be considered as elements in $K$. In $K, \bar{A}+(\bar{B}+\bar{C})=(\bar{A}+\bar{B})+\bar{C}$ $=\sum_{i=1}^{k} z_{i} t^{(i)}+x_{k}$, where each $z_{i}$ is in $M$, each $t^{\mu(i)}$ is in $G, k$ is any positive integer, each $\mu(i)$ is in $\Delta$, and $V\left(x_{k}\right)>\mu(k)$.

It follows from 2.1 that if $\alpha$ is any element in $\Gamma$, there are at most a finite number of $\mu(i)$ that are less than $\alpha$. Hence there is an integer $k$ such that $\mu(k)=\delta$. (Without condition 2.1, we could not be sure that this would be so.) But the coefficients $z_{i}$ are determined in the same manner as they would be if the addition were taking place in $M\left(t^{\Gamma}\right)$. Hence $z_{k}=r_{\delta}=p_{\delta}$.

Similarly it can be shown that $d_{\delta}=s_{\delta}$ and that $n_{\delta}=y_{\delta}$.

We now shall show that if $A=\sum a_{\alpha} t^{\alpha}, R=\sum r_{\alpha} t^{\alpha}, \alpha \in \phi$, are any two elements in $M\left(t^{\mathbf{\Gamma}}\right)$, then the equation $A+B=R$ has a solution in $M\left(t^{\mathbf{\Gamma}}\right)$.

Let $\Delta$ be the set of all elements in the form $\alpha+\gamma$, for all $\alpha$ in $\phi$ and all $\gamma$ in $\theta$. Then $A=\sum a_{\delta} t^{\delta}, R=\sum r_{\delta} t^{\delta}, \delta \in \Delta$.

Let $\epsilon$ be the smallest element in $\Delta$. Let $b_{\epsilon}=\left(r_{\epsilon}-a_{\epsilon}\right)^{\prime}, B[\epsilon]=B\left[a_{\epsilon}+b_{\epsilon}\right]$, and $c_{\epsilon}=c(B[\epsilon], \epsilon)$.

Assume that $b_{\rho}$ has been chosen for all $\rho$ in $\Delta$ less than $\delta$ in $\Delta$, and that the corresponding elements, $B[\rho]$ and $c_{\rho}$, have been determined.

Then $b_{\delta}=\left(r_{\delta}-\left[a_{\delta}+\sum_{\rho} c_{\rho}\right]\right)^{\prime}$, where $\rho$ runs over all elements in $\Delta$ having the property $V(B[\rho])+\rho=\delta . B[\delta]=B\left[a_{\delta}+b_{\delta}+\sum c_{\rho}\right]$ and $c_{\delta}=c(B[\delta], \delta)$.

Let $B=\sum b_{\delta} t^{\delta}, \delta \in \Delta$. Let $A+B=D=\sum d_{\delta} t^{\delta}, \delta \in \Delta$. Then $d_{\epsilon}=\left(a_{\epsilon}+b_{\epsilon}\right)^{\prime}$, and $d_{\epsilon}-r_{\epsilon}=\left(a_{\epsilon}+\left[r_{\epsilon}-a_{\epsilon}\right]^{\prime}\right)^{\prime}-r_{\epsilon}=0$.

Assume that $d_{\rho}=r_{\rho}$ for all $\rho$ in $\Delta$ less than $\delta$ in $\Delta . d_{\delta}=\left(a_{\delta}+b_{\delta}+\sum c_{\rho}\right)^{\prime}$ and $d_{\delta}-r_{\delta}=\left[a_{\delta}+\sum c_{\rho}+\left(r_{\delta}-\left[a_{\delta}+\sum c_{\rho}\right]\right)^{\prime}\right]^{\prime}-r_{\delta}=0$. Hence $R=D$, that is, $A+B$ $=R$.

We now show that each element $A=\sum a_{\alpha} t^{\alpha} \neq 0, \alpha \in \phi$, in $M\left(t^{\Gamma}\right)$ has an inverse in $M\left(t^{\mathrm{r}}\right)$. Let $\sigma$ be the smallest element in $\phi$. We may assume that $a_{\sigma} \neq 0$.

Let $x$ and $y$ be any two elements in $M$, and let $\alpha$ and $\beta$ be any two elements in $\Gamma$. Then if $x y \neq 0$, let $m$ be the element in $M$ that is in the same residue class in $\Omega$ as the element $y / x c\left(t^{\alpha}, \beta\right)$, and let $r(x, y, \alpha, \beta)=m / y$. If $x y=0$, let $r(x, y, \alpha, \beta)=0$.

Let $\Lambda$ be the set of all finite sums of elements of the form $\alpha+\gamma-\sigma$, for all $\alpha$ in $\phi$ and all $\gamma$ in $\theta$. Each element in $\Lambda$ is positive or 0 . The set of all elements of the form $\alpha+\gamma-\sigma$ is well-ordered. Hence [3, Theorem 3] implies that $\Lambda$ is well-ordered.

Let $C=1=c_{0} t^{0}$. We shall use a method similar to one used by Hahn [4] 
to construct $B$ so that $A B=C$.

Let $\beta$ be the smallest positive element in $\Lambda$. Let $B_{\beta}=c_{0} r\left(a_{\sigma}, c_{0}, 0-\sigma, \sigma\right) t^{0-\sigma}$. Let $C_{\beta}=C-B_{\beta} A=c_{0} t^{0}-\left(a_{\sigma} c_{0} c\left(t^{0-\sigma}, \sigma\right) r\left(a_{\sigma}, c_{0}, 0-\sigma, \sigma\right)\right)^{\prime} t^{0}+\cdots=c_{\beta} t^{\beta}+\cdots$.

Assume that for every $\rho$ in $\Lambda$ less than $\lambda$ in $\Lambda, B_{\rho}$ has been chosen so that $B_{\rho}=\sum_{\mu} c_{\mu} r\left(a_{\sigma}, c_{\mu}, \mu-\sigma, \sigma\right) t^{\mu-\sigma}$, where $\mu$ is in $\Lambda, 0 \leqq \mu<\rho$, and $C_{\rho}=C-B_{\rho} A$ $=c_{\rho} t^{\rho}+\cdots$.

Then $B_{\lambda}=\sum_{\rho} c_{\rho} r\left(a_{\sigma}, c_{\rho}, \rho-\sigma, \sigma\right) t^{\rho-\sigma}, 0 \leqq \rho<\lambda . C_{\lambda}=C-B_{\lambda} A=c_{r} t^{\tau}+\cdots$, where $\tau$ is in $\Lambda$.

Let $B_{\lambda}=B_{\rho}+B_{\rho, *}$ where $B_{\rho, *}=\sum_{\mu} c_{\mu} r\left(a_{\sigma}, c_{\mu}, \mu-\sigma, \sigma\right) t^{\mu-\sigma}$, where $\mu$ is in $\Lambda, \rho \leqq \mu<\lambda$.

Then $C_{\lambda}=C-\left(B_{\rho}+B_{\rho, *}\right) A=C-B_{\rho} A-B_{\rho, *} A=C_{\rho}-B_{\rho, *} A=\left(c_{\rho} t^{\rho}+\cdots\right)$ $-\left(c_{\rho} t^{\rho}+\cdots\right)$.

Hence $\tau>\rho$ for all $\rho$ in $\Lambda$ less than $\lambda$ in $\Lambda$. And so $\tau$ can be taken equal to $\lambda$. Let $B=\sum_{\lambda} c_{\lambda} r\left(a_{\sigma}, c_{\lambda}, \lambda-\sigma, \sigma\right) t^{\lambda-\sigma}, \lambda \in \Lambda$. Then $C-B A=0$.

And so it is seen that $M\left(t^{\Gamma}\right)$ is a field.

Let $A=\sum a_{\alpha} t^{\alpha}, \alpha \in \phi$, be any element in $M\left(t^{\mathrm{\Gamma}}\right)$, and assume that $a_{\sigma}$, where $\sigma$ is the smallest element in $\phi$, is not 0 . Let $V^{\prime}(A)=\sigma$. It is seen that $V^{\prime}$ constitutes a valuation of $M\left(t^{\Gamma}\right)$ and that corresponding to this valuation, $\Gamma$ is the value group and $\Omega$ the residue class field.

It is noted that the ordinary power series field constructed by Hahn [4] and investigated by Krull [5] is a special case of $M\left(t^{\mathbf{r}}\right)$, where $C$ consists solely of 1 , and $L$ consists solely of 0 .

It can be proved directly that $M\left(t^{\mathbf{\Gamma}}\right)$ is maximal with respect to the valuation $V^{\prime}$. But instead, we shall prove another theorem of which $M\left(t^{\Gamma}\right)^{\text {'s }}$ being maximal will be a corollary.

Let $J$ be the set of all elements, $a$, in $K$ such that $V(a) \geqq 0$. For $a$ in $J$, let $H(a)$ be the residue class in $\Re$ to which $a$ belongs. Let $\Re\left(x^{\Gamma}\right)$ be the power series fleld constructed by Hahn [4], consisting of all formal power series of the form $\sum_{\alpha} H\left(b_{\alpha}\right) x^{\alpha}, \alpha \in \phi$, where the $b_{\alpha}$ are in $J$, and $\phi$ is a well-ordered subset of elements in $\Gamma$. Krull [5, Theorem 25] has shown that there is a oneto-one map of $K$ into $\Re\left(x^{\Gamma}\right)$ such that:

1. If $\bar{f}$ in $\Re\left(x^{\Gamma}\right)$ corresponds to an element in $K$, then each segment (abschnitt) of $\bar{f}$ corresponds to an element in $K$.

2. If $f$ and $h$ are any two elements in $K, \bar{f}$ and $\bar{h}$ the corresponding elements in $\Omega\left(x^{\Gamma}\right)$, and if $\bar{f}-\bar{h}=H(a) x^{\alpha}+\cdots$, then $V\left(f-h-a^{\prime} t^{\alpha}\right)>V(f-h)$ (where $a^{\prime}$ is the correspond of $a$ in $M$ ).

3. $a^{\prime} t^{\alpha}$ corresponds to $H\left(a^{\prime}\right) x^{\alpha}$, where $a^{\prime}$ is in $M$ and $t^{\alpha}$ is in $G$.

THEOREM 2.6. Let $K$ be mapped one-to-one into $\Re\left(x^{\Gamma}\right)$ so that the map satisfies (1), (2), and (3). Then $K$ is maximal if, and only if, every element in $\Re\left(x^{\mathbf{\Gamma}}\right)$ is the correspond of some element in $K$ under this map.

Proof. We first assume that every element in $\Re\left(x^{\Gamma}\right)$ is the correspond of some element in $K$. Let $\left\{a_{\rho}\right\}$, where $\rho$ runs over all ordinals less than a given 
ordinal $\sigma$, be any pseudo-convergent set $\left({ }^{2}\right)$ in $K$. Let $a_{\rho}$ correspond to $\bar{a}_{\rho}=\sum H\left(a_{\alpha(\rho)}\right) x^{\alpha(\rho)}$ in $\Omega\left(x^{\mathbf{\Gamma}}\right)$. Let $\tau, \mu$, and $\nu$ be any three ordinals such that $\sigma>\tau>\nu, \sigma>\mu>\nu$. Then $V\left(a_{\nu}-a_{\tau}\right)=V\left(a_{\nu}-a_{\mu}\right)=\beta(\nu)$, by [1, Lemma 2]. Hence by (2), $H\left(a_{\alpha(v)}\right)=H\left(a_{\alpha(r)}\right)$ and $\alpha(\nu)=\alpha(\tau)$ for all values $\alpha(\nu)$ that are less than $\beta(\nu)$, for all ordinals $\tau$ that are greater than $\nu$ and less than $\sigma$.

Let $\bar{f}=\sum H\left(a_{\beta}\right) x^{\beta}$, where the $a_{\beta}$ are in $J$, and where $H\left(a_{\beta}\right)=H\left(a_{\alpha(v)}\right)$ and $\beta=\alpha(\nu)$ for every $\beta$ that is less than $\beta(\nu)$, for every ordinal $\nu$ less than $\sigma$. $\bar{f}$ is in $\Omega\left(x^{\Gamma}\right)$ and so corresponds to an element $f$ in $K$. It follows from (2) that $V\left(f-a_{\nu}\right) \geqq \beta(\nu)$ for every ordinal $\nu$ less than $\sigma$.

$$
V\left(f-a_{\nu}\right)=V\left[\left(f-a_{\tau}\right)-\left(a_{\nu}-a_{\tau}\right)\right]=V\left(a_{\nu}-a_{\tau}\right)=\beta(\nu)
$$

when $\sigma>\tau>\nu$. Hence $f$ is a limit of the sequence $\left\{a_{p}\right\}$, and so $K$ is maximal by $[1$, Theorem 4$]$.

Now we assume that $K$ is maximal. Suppose that $\bar{c}=\sum H\left(a_{\alpha}\right) x^{\alpha}, \alpha \in \phi$, in $\Omega\left(x^{\mathbf{r}}\right)$, is not the correspond of an element in $K$. Let $\epsilon$ be the smallest element in $\phi$. (3) implies that $H\left(a_{e}\right) x^{\bullet}$ is the correspond of an element in $K$. Let $\beta$ be the smallest element in $\phi$ such that $\bar{b}=\sum H\left(a_{\alpha}\right) x^{\alpha}, \epsilon \leqq \alpha<\beta, \alpha \in \phi$, is not the correspond of an element in $K$.

Let there be a largest element $\delta$ in $\phi$ that is less than $\beta$. Then $\bar{d}=\sum H\left(a_{\alpha}\right) x^{\alpha}, \epsilon \leqq \alpha<\delta$, is the correspond of an element $d$ in $K$. Let $f=d+a_{\delta}^{\prime} t^{\delta}$. Let $\bar{f}$ be the correspond in $\Omega\left(x^{\mathbf{T}}\right)$ of $f . f-d=a_{\delta}^{\prime} t^{\delta}$. Therefore it follows from (2) that $\bar{f}-\bar{d}=H\left(a_{\delta}\right) x^{\delta}+\cdots$. This implies that $\bar{b}$ is a segment of $\bar{f}$. Hence (1) implies that $b$ corresponds to an element in $K$.

Now let there be no largest element in $\phi$ that is less than $\beta$. Let $\alpha(\rho)$ be the element in $\phi$ such that the order type of the elements in $\phi$ less than $\alpha(\rho)$ is $\rho$. Let $\beta=\alpha(\sigma)$. Let $\left\{a_{\rho}\right\}$, where $\rho$ runs over all ordinals less than $\sigma$, be a sequence in $K$ such that $\bar{a}_{\rho}=\sum H\left(a_{\alpha}\right) x^{\alpha}, \epsilon \leqq \alpha<\alpha(\rho), \alpha \in \phi$. When $\sigma>\tau>\nu$, (2) implies that $V\left(a_{\nu}-a_{\tau}\right)=\alpha(\nu)$. Hence when $\sigma>\tau>\nu, \sigma>\mu>\nu, \sigma>\lambda>\nu$, then $V\left(a_{\nu}-a_{\tau}\right)$ $<V\left(a_{\mu}-a_{\lambda}\right)$. Therefore $\left\{a_{\rho}\right\}$ is a pseudo-convergent set and has a limit $r$ in $K$, by [1, Theorem 4]. Let $\bar{r}$ be the correspond in $\Re\left(x^{\mathbf{r}}\right)$ of $r . \bar{r}=\sum H\left(c_{\alpha}\right) x^{\alpha}$. (2) implies that $H\left(c_{\alpha}\right)=H\left(a_{\alpha}\right)$ for all $\alpha$ less than $\beta$. Hence $\bar{r}$ has $b$ as a segment. And so it is seen by (1) that $b$ is the correspond of an element in $K$.

Corollary 2.7. Any two fields with valuations that are maximal and that have the same residue class field $\AA$ and the same value group $\Gamma$ have the same cardinal number (that is, the cardinal number of the field $\AA\left(x^{\mathbf{\Gamma}}\right)$ ).

COROLlaRY 2.8. The field $M\left(t^{\mathbf{\Gamma}}\right)$ is maximal.

Proof. $M\left(t^{\Gamma}\right)$ can be mapped upon the field $\Re\left(x^{\mathbf{\Gamma}}\right)$ by letting $\sum a_{\alpha} t^{\alpha}$ in $M\left(t^{\Gamma}\right)$ correspond to $\sum H\left(a_{\alpha}\right) x^{\alpha}$ in $\Re\left(x^{\Gamma}\right)$. This map satisfies (1), (2), and (3).

3. Structure of maximal fields. For convenient reference, we state here the pair of conditions that Kaplansky [1] called and that we shall refer to as

(2) For a definition of pseudo-convergence, see [1]. 
hypothesis A. Let the characteristic of $\Omega$ be $p$.

1. Any equation of the form $x^{p^{n}}+a_{1} x^{p^{n-1}}+\cdots+a_{n-1} x^{p}+a_{n} x+a_{n+1}=0$ with coefficients in $\Omega$ has a root in $\Omega$.

2. The value group $\Gamma$ satisfies $\Gamma=p \Gamma$.

If the characteristic of $\Omega$ is 0 , hypothesis $A$ is to be considered vacuous.

LEMMA 3.1. 1. If $M$ and $G$ can be chosen so that 2.1 is satisfied, and if $\Omega$ and $\Gamma$ satisfy hypothesis $\mathrm{A}$, then $K$ is analytically isomorphic to a subfield of $M\left(t^{\mathbf{\Gamma}}\right)$.

2. If $\Gamma$ is archimedean and discrete (that is, essentially the additive group of integers), then $K$ is analytically isomorphic to a subfield of $M\left(t^{\Gamma}\right)$.

Proof. It is seen that in (2), 2.1 is satisfied for any choice of $M$ and $G$.

Let $M$ and $G$ be chosen in both (1) and (2) so that 2.1 is satisfied. Let $\bar{K}$ be the complete (topologically) closure $\left(^{3}\right)$ of $K$. Let $D$ be the set of all elements in $\bar{K}$ of the form $\sum_{i=1}^{k} a_{i} t^{(i)}+x_{k}$, where the $a_{i}$ are in $M$, the $t^{\alpha(i)}$ in $G, \alpha(i)<\alpha(j)$ for $i<j, k$ is any positive integer, $V\left(x_{k}\right)>\alpha(k)$, and the $\alpha(i)$ are such that at most a finite number of them are less than any given element in $\Gamma$.

Let $A=\sum_{i=1}^{k} a_{i} t^{\alpha(i)}+x_{k}$ and $B=\sum_{i=1}^{k} b_{i} t^{\alpha(i)}+\dot{y}_{k}$ be any two elements in $D . A+B=\sum_{i=1}^{k} c_{i} \beta^{(i)}+z_{k}$, where the $c_{i}$ are in $M$, the $t^{\beta(i)}$ are in $G$, and $V\left(z_{k}\right)>\beta(k)$. The coefficients $c_{i}$ are determined by the method of 2.2 , and each $\beta(i)$ is of the form $\alpha(j)+\gamma$, where $\gamma$ is in $\theta$, and $1 \leqq j<\infty$. It follows from 2.1 that at most a finite number of the $\beta(i)$ are less than any given element in $\Gamma$. Hence $A+B$ is in $D$. Similarly it can be shown that $A B$ is in $D$.

Now let $A^{\prime}=\sum_{i=1}^{\infty} a_{i} t^{\alpha(i)}$ and $B^{\prime}=\sum_{i=1}^{\infty} b_{i} t^{\alpha(i)}$ be considered as elements in the field $M\left(t^{\Gamma}\right)$. Let $C^{\prime}=\sum c_{\beta} t^{\beta}, \beta \in \phi$, be the element $B^{\prime}-A^{\prime}$ in $M\left(t^{\Gamma}\right)$. Each element in $\phi$ is of the form $\alpha(i)+\gamma$, where $\gamma$ is in $\theta$, and $1 \leqq i<\infty$. Hence at most a finite number of the elements in $\phi$ are less than any given element in $\Gamma$. Therefore $C^{\prime}=\sum_{i=1}^{\infty} c_{i} t^{(i)}$. Consider the sequence of elements in $\bar{K}$ of the form $\sum_{i=1}^{y} c_{i} t^{\beta(i)}$, for $1 \leqq j<\infty$. This is a Cauchy sequence in $\bar{K}$ and so has a limit $C$ in $\bar{K} . C=\sum_{i=1}^{k} \cdot c_{i} i^{\beta(i)}+z_{k}$, where $k$ is any positive integer and $V\left(z_{k}\right)>\beta(k) . C$ is in $D$, and it is seen that $A+C=B$.

And so $D$ is an integral domain. Let $D^{\prime}$ be the set of all elements in $M\left(t^{\mathbf{r}}\right)$ of the form $\sum_{i=1}^{\infty} a_{i} t^{\alpha(i)}$, where at most a finite number of the $\alpha(i)$ are less than any given element in $\Gamma$.

Let $A=\sum_{i=1}^{k} a_{i} t^{(i)}+x_{k}$ in $D$ correspond to $A^{\prime}=\sum_{i=1}^{\infty} a_{i} t^{\alpha(i)}$ in $D^{\prime}$. This is a one-to-one correspondence, since $\bar{K}$ is complete. This correspondence is seen to be an analytic isomorphism between $D$ and $D^{\prime}$. Hence the quotient field $E$ of $D$ is analytically isomorphic to $E^{\prime}$, the quotient field of $D^{\prime} . M\left(t^{\mathrm{r}}\right)$ is an immediate maximal extension of $E^{\prime}$. Any immediate maximal extension of $\bar{K}$ is an immediate maximal extension of $E$. In (1), [1, Theorem 5] implies that any such maximal extension is analytically isomorphic to $M\left(t^{\Gamma}\right)$. In (2),

( $\left.{ }^{3}\right) \bar{K}$ is the field $\mathfrak{A} \bmod \mathfrak{R}$, where $\mathfrak{A}$ is the ring of all ordinary Cauchy sequences in $K$ and $\mathfrak{R}$ is the ideal of all null sequences in $K$. That $\bar{K}$ is unique up to analytic isomorphism can be proved in the same manner as it is proved for the case where $\Gamma$ is archimedean. 
$E^{\prime}$ is the field $M\left(t^{\Gamma}\right)$, and $E$ is the field $\bar{K}$. Therefore $K$ is analytically isomorphic to a subfield of $M\left(t^{\mathbf{\Gamma}}\right)$.

Lemma 3.2. Let $K$ be maximal. If the characteristic $p$ of $\Omega$ is not equal to 0 , let every element in $K$ have a pth root in $K$. If $\Gamma^{\prime}$ is a subgroup of $\Gamma$ such that there exists a subfield of $K$ that under the valuation induced by $V$ has $\Gamma^{\prime}$ as its value group, then $K$ has a subfield that under the valuation induced by $V$ has $\Gamma^{\prime}$ as its value group and $\AA$ as its residue class field.

Proof. $\left({ }^{4}\right)$. Let $T$ be a subfield of $K$ that under the valuation induced by $V$ has $\Gamma^{\prime}$ as its value group; let $\mathfrak{T}$ be the corresponding residue class field.

Our hypothesis implies that $\Omega$ is perfect. Hence $\Omega$ may be built up from $\mathfrak{T}$ by successive transcendental adjunctions, $p$ th root adiunctions (if $p \neq 0$ ), and joins of such adjunctions, and separable algebraic adjunctions. We shall build up our required subfield of $K$ through a succession of fields paralleling adjunctions in $\Omega$ over $\mathfrak{T}$. It suffices to consider a single transcendental, $p$ th root (if $p \neq 0$ ), and separable algebraic adjunction.

Let $S$ be a subfield of $K$ that contains $T$, and let $\subseteq$ be the residue class field and $\Gamma^{\prime}$ the value group of $S$ with respect to $V$. Let $x$ be any element in $\Re$ that is transcendental over $\mathfrak{S}$. Let $y$ be an element in $K$ that is in the residue class $x$. Then $y$ is transcendental over $S$. Let $r=a_{0}+a_{1} y+\cdots$ $+a_{n-1} y^{n-1}$ be any element in the domain $S[y]$. It can be verified that $V(r)=$ minimum $\left\{V\left(a_{i}\right)\right\}$, for $0 \leqq i \leqq n-1$; otherwise, $x$ would have to be algebraic over $\mathfrak{S}$. And so the field $S(y)$ has $\Gamma^{\prime}$ as its value group and $\subseteq(x)$ as its residue class field.

Let $x$ be a $p$ th root, if $p \neq 0$, of an element in $\mathfrak{S}$ or separably algebraic over $\mathfrak{S}$, and in $\Omega$. Let $g(z)$ be the monic irreducible polynomial in $\mathfrak{S}$ of which $x$ is a zero. Choose in $S$ any monic polynomial $G(z)$ of the same degree as $g(z)$ and with coefficients representative of the coefficients of $g(z)$; if the coefficient of $z^{i}$ in $g(z)$ is 0 , let the coefficient of $z^{i}$ in $G(z)$ be 0 . If $x$ is separable over S, the Hensel-Rychlik reducibility theorem [5, Theorem 12] implies that $K$ contains a root $y$ of $G(z)=0$ in the residue class $x$. If $x$ is the pth root of an element in $\subseteq$, then by hypothesis, $G(z)=0$ has a root $y$ in $K$ that is in the residue class $x$. Since $g(z)$ is irreducible, $G(z)$ is irreducible. As before, let $r$ be any element in $S(y)$. It can be verified that $V(r)=\operatorname{minimum}\left\{V\left(a_{i}\right)\right\}$, for $0 \leqq 1$ $\leqq n-1$; otherwise $x$ would satisfy a polynomial over $\subseteq$ of degree less than the degree of $g(z)$. Hence $S(y)$ has $\Gamma^{\prime}$ as its value group and $\mathfrak{S}(x)$ as its residue class field.

Now let $\Delta_{i}, 0 \leqq i \leqq n$, be a finite set of isolated subgroups of $\Gamma$ such that $\Delta_{0}=0, \Delta_{n}=\Gamma$, and $\Delta_{i-1}$ is a subgroup of $\Delta_{i}$ for $0<i \leqq n$. Let $B$ be the valuation ring of $K$, that is, the set of all elements $a$ in $K$ such that $V(a) \geqq 0$. Let $P_{i}$ be the prime ideal corresponding to $\Delta_{i}$, that is, $P_{i}$ contains all $a$ in $B$ such that

(4) The proof of this lemma comes directly from the proof of [1, Theorem 7] which in turn depends upon the proof of [6, Theorem 3]. The argument is merely consolidated here. 
$V(a)$ is not in $\Delta_{i}$. Let $B_{P_{i}}$ be the set of all elements in $K$ of the form $a / b$, where $a$ and $b$ are in $B$, but $b$ is not in $P_{i}$. Let $\Omega_{i}$ be the field $B_{P_{i}} / P_{i}$. Then $\Omega_{n}=K, \Omega_{0}=\Omega$.

Then by the Aufgespaltungsprozess $\left[5\right.$, p. 173], there is a valuation $V_{i}$ of $\Omega_{i}$ with residue class field $\Omega_{i-1}$, and value group $\Delta_{i} / \Delta_{i-1}$, for $0<i \leqq n$.

LEMma 3.3. If $K$ is maximal with respect to $V$, then $\Omega_{i}$ is maximal with respect to $V_{i}$, for $0<i \leqq n$.

Proof. It will suffice if we prove the theorem for the case where $n=2$. We first show that $K=\Omega_{2}$ is maximal with respect to $V_{2}$. Let $\left\{a_{\rho}\right\}$ be any pseudoconvergent set in $\Omega_{2}$ with respect to $V_{2}$. Then $V_{2}\left(a_{\sigma}-a_{\rho}\right)<V_{2}\left(a_{\tau}-a_{\sigma}\right)$ for all $\rho<\sigma<\tau$. Therefore $V\left(a_{\sigma}-a_{\rho}\right)<V\left(a_{\tau}-a_{\sigma}\right)$ for all $\rho<\sigma<\tau$. Hence $\left\{a_{\rho}\right\}$ is a pseudo-convergent set with respect to $V$ and so has a limit $a$ in $K$, by [1, Theorem 4]. $V\left(a-a_{\rho}\right)=V\left(a_{\sigma}-a_{\rho}\right)=\gamma_{\rho}$, for $\sigma>\rho$, for all $\rho$. And so $V_{2}\left(a-a_{\rho}\right)$ $=V_{2}\left(a_{\sigma}-a_{\rho}\right)$, for $\sigma>\rho$, for all $\rho$. Therefore $a$ is a limit of $\left\{a_{\rho}\right\}$ with respect to $V_{2}$, and so $K$ is maximal with respect to $V_{2}$, by [1, Theorem 4].

We now show that $\Omega_{1}$ is maximal with respect to $V_{1}$. Let $\left\{a_{\rho}\right\}$ be any pseudo-convergent set in $\Omega_{1}$. Let $\bar{a}_{\rho}$ in $B_{P_{1}}$ be a representative of $a_{\rho}$, for all $\rho$. $V\left(\bar{a}_{\rho}-\bar{a}_{\sigma}\right)=V_{1}\left(a_{\rho}-a_{\sigma}\right)$ for all $\rho$ and $\sigma$. Hence $\left\{\bar{a}_{\rho}\right\}$ is a pseudo-convergent set in $K$ with respect to $V$, and so has a limit $\bar{a}$ in $K$. If 0 is a limit of $\left\{\bar{a}_{\rho}\right\}$ with respect to $V$, then 0 is a limit of $\left\{a_{\rho}\right\}$ with respect to $V_{1}$. If 0 is not a limit of $\left\{\bar{a}_{\rho}\right\}$, then $V(\bar{a})=V\left(\bar{a}_{\rho}\right)$ for all $\rho$ greater than or equal to some ordinal $\lambda$, by [1, Lemma 1]. Hence, in this case, $V(\bar{a})$ is in $\Delta_{1} / \Delta_{0}=\Delta_{1}$, and $\bar{a}$ is in $B_{P_{1}}$. Let $a$ be the residue class in $\Omega_{1}$ to which $\bar{a}$ belongs. It is seen that $a$ is a limit of $\left\{a_{\rho}\right\}$ with respect to $V_{1}$. Therefore $\Omega_{1}$ is maximal with respect to $V_{1}$.

LEMMA 3.4. Let the characteristic of $\Omega$ be $p \neq 0$, let the characteristic of $K$ be 0 , and let $j$ be the smallest integer such that $\Delta_{j}$ contains $V(p)$. Then the characteristic of $\Omega_{i}$ is 0 for $n \geqq i \geqq j$, and is $p$ for $j>i \geqq 0$.

Proof. Let $c_{i}$ be an element in $B_{P_{i}}$, not in $P_{i}$, and let $q_{i}$ be the characteristic of $\Omega_{i}$, for $0 \leqq i \leqq n$. Since $q_{i} c_{i}$ is in $P_{i}$, it follows that $q_{i}$ is in $P_{i}$. Hence $q_{i}$ is 0 or $p$. If $i<j$, then $V(p)$ is not in $\Delta_{i}$, and so $p$ is in $P_{i}$. If $i \geqq j$, then $V(p)$ is in. $\Delta_{i}$, and so $p$ is. not in $P_{i}$.

Let $M_{i}\left(t_{i} / \Delta_{i}-1\right), 0<i \leqq n$, be defined for $\Omega_{i}$ with respect to $V_{i}$ in the same manner as $M\left(t^{\mathrm{r}}\right)$ is defined for $K$ with respect to $V$.

TheOREM 3.5. Let $K$ be maximal and let $\Omega$ and $\Gamma$ satisfy hypothesis A. Let $\Gamma=\Delta_{2}$. If the characteristic $p$ of $\Omega$ is the same as the characteristic of $K$, let $\Delta_{2}=\Delta_{1}$. If the characteristic of $K$ is 0 and $p \neq 0$, let $\Delta_{1}$ be the isolated subgroup generated by $V(p)$. Then $K=\Omega_{2}$ is analytically isomorphic to a generalized power series field $M_{2}\left(t^{\Delta_{2} / \Delta_{1}}\right)$ with respect to $V_{2}$, and $M_{2}=\Omega_{1}$ is analytically isomorphic to a generalized power series field $M_{1}\left(t^{\Delta_{1} / \Delta_{0}}\right)$ with respect to $V_{1}$. 
Proof. $\left({ }^{5}\right)$. Let $q$ be the characteristic of $K$. If $p=q$, then $M_{\mathbf{2}}\left(t^{\Delta_{2} / \Delta_{1}}\right)=\Omega_{1}$ $=\Omega_{2}=K$, and $\Delta_{2} / \Delta_{1}$ consists of the one element, 0 . If $p \neq 0$ and $q=0$, then by Lemma 3.4, the characteristic of $\Omega_{1}$ is 0 . By Lemma 3.3, $K=\Omega_{2}$ is maximal with respect to $V_{2}$. Hence by [1, Theorem 6 ], $K$ is analytically isomorphic to $M_{2}\left(t^{\Delta_{2} / \Delta_{1}}\right)$ with respect to $V_{2}$, and $M_{2}=\Omega_{1}$.

If $p=q, \Gamma=\Delta_{1}=\Delta_{1} / \Delta_{0}$ and $\Omega_{0}=\Omega$ satisfy hypothesis $A$. Hence by [ 1 , Theorem 6], $\Omega_{1}=K$ is analytically isomorphic to $M_{1}\left(t^{\Delta_{1} / \Delta_{0}}\right)$ with respect to $V_{1}$.

Now assume that $p \neq 0$ and $q=0$. Then since $\Gamma=p \Gamma$, it follows that $\Delta_{1} / \Delta_{0}=\Delta_{1}=p \Delta_{1}$. Hence $\Delta_{1} / \Delta_{0}$ and $\Omega_{0}$ satisfy hypothesis A.

The prime subfield of $\Omega_{1}$ has the discrete group $\Delta$ that is generated by $V_{1}(p)$ as its value group with respect to the valuation induced by $V_{1}$. [1, Lemma 15] implies that every element in $\Omega_{1}$ has a $p$ th root in $\Omega_{1}$. Therefore 3.2 implies that $\Omega_{1}$ has a subfield that under the valuation induced by $V_{1}$ has $\Delta$ as its value group and $\Re=\Omega_{0}$ as its residue class field. Let $\Re$ be the unique complete closure of this subfield. Then $\Re$ is a subfield of $\Omega_{1}$.

[7, Theorem 5] implies that $\Re$ contains a system $M_{1}$ of representatives of the residue classes in $\Omega_{0}$ such that if $a, b$, and $c$ are the representatives in $M_{1}$ of $\mathfrak{a}, \mathfrak{b}$, and $\mathfrak{c}$ respectively in $\Omega_{0}$, then $\mathfrak{a} \mathfrak{b}=\mathfrak{c}$ implies that $a b=c$.

We can select a system $G_{1}$ of representatives in $\Omega_{1}$ of the values in $\Delta_{1}$ such that $t^{V_{1}(p)}$ is in $\Re$ and such that if $t^{\alpha}$ and $t^{\beta}$ are elements in $G_{1}$ of values $\alpha$ and $\beta$ respectively, then $c\left(t^{\alpha}, \beta\right)=t^{\alpha} t^{\beta} / t^{\alpha+\beta}$ is in $M_{1}$. The proof of this fact is exactly the same as the proof of $[1$, Lemma 13] taken in conjunction with [1, Lemma 15].

Our result now follows from 3.1.

THEOREM 3.6. Let $K$ be maximal and let $\Gamma$ be discrete in the sense of Kaplansky [2]. Let $\Gamma=\Delta_{3}$. If the characteristic $p$ of $\Omega$ is the same as the characteristic of $K$, let $\Delta_{3}=\Delta_{2}=\Delta_{1}$. If the characteristic of $K$ is 0 and $p \neq 0$, let $\Delta_{2}$ be the isolated subgroup generated by $V(p)$, and let $\Delta_{1}$ be.the largest $\left(^{6}\right)$ isolated subgroup contained in $\Delta_{2}$. Then $K=\Omega_{3}$ is analytically isomorphic to a generalized power series field $M_{3}\left(t_{3} / \Delta_{2}\right)$ with respect to $V_{3} ; M_{3}=\Omega_{2}$ is analytically isomorphic to $\dot{a}$ generalized power series field $M_{2}\left(t^{\Delta_{2} / \Delta_{1}}\right)$ with respect to $V_{2}$; and $\Omega_{1}$ (of whose elements $M_{2}$ is a set of representatives in $\Omega_{2}$ ) is analytically isomorphic to a generalized power series field $M_{1}\left(t^{\Delta_{1} / \Delta_{0}}\right)$ with respect to $V_{1}$, where $M_{1}=\Omega$.

Proof. Let $q$ be the characteristic of $K$. If $p=q$, the results follow from [2, Theorem].

If $q=0$ and $p \neq 0$, then Lemma 3.4 implies that the characteristic of $\Omega_{2}$ is 0. By Lemma 3.3, $\Omega_{3}$ is maximal with respect to $V_{3} . \Delta_{3} / \Delta_{2}$ is discrete since $\Gamma=\Delta_{3}$ is discrete. Hence [2, Theorem] implies that $\Omega_{3}$ is analytically iso-

(5) A suggestion by Irving Kaplansky to the writer led to the formulation of this theorem for the case where the characteristic of $K$ is not $p$ and $\Delta_{2} \neq \Delta_{1}$.

(b) It can be verified easily that the subgroup consisting of all elements $\alpha$ in $\Gamma$ such that $n \alpha<V(p)$ for every integer $n$ is the largest isolated subgroup contained in $\Delta_{2}$. 
morphic to $M_{3}\left(t^{\Delta_{3} / \Delta_{2}}\right)$ with respect to $V_{2}$, and $M_{3}=\Omega_{2}$. By 3.4, the characteristic of $\Omega_{1}$ is $p . \Delta_{2} / \Delta_{1}$ is discrete and archimedean. And so it follows from 3.1 that $\Omega_{2}$ is analytically isomorphic to $M_{2}\left(t^{\Delta_{2} / \Delta_{1}}\right)$. Since the characteristic of $\Omega_{1}$ is $p$ and since $\Omega_{1}$ is maximal with respect to $V_{1},[2$, Theorem ] implies that $\Omega_{1}$ is analytically isomorphic to $M_{1}\left(t^{\Delta_{1} / \Delta_{0}}\right)$ with respect to $V_{1}$, where $M_{1}$ $=\Omega_{0}=\Omega$.

COROLlary 3.7. Let $K$ be maximal. Then $K$ is analytically isomorphic to a generalized power series field, $M\left(t^{\mathrm{r}}\right)$, with respect to $V$, if any one of the following conditions is met:

1. $\Omega$ and $\Gamma$ satisfy hypothesis $A$, and if the characteristic $p$ of $\Omega$ is different from the characteristic of $K, \Gamma$ is the isolated subgroup generated by $V(p)$.

2. $\Gamma$ is archimedean, and $\Omega$ and $\Gamma$ satisfy hypothesis $\mathrm{A}$.

3. $\Gamma$ is discrete, and the characteristic of $\Omega$ is the same as that of $K$.

4. $\Gamma$ is archimedean and discrete.

Corollary 3.8. Let $\Delta_{2}$ and $\Delta_{1}$ be defined as in 3.5. Then $K$ is analytically isomorphic to a subfield of a generalized power series field with respect to $V_{2}$, and the coefficient field of this power series field is analytically isomorphic to a generalized power series field with respect to a valuation that is an extension of $V_{1}$.

COROLLARY 3.9. Every field with a valuation $V$ on an archimedean value group is analytically isomorphic to a subfield of a suitable generalized power series field with respect to $V$.

The last two corollaries follow from the fact that a field with a valuation can be imbedded in an overfield with a valuation that is an extension of the given valuation and with value group and residue class field satisfying hypothesis $A$, by $[8$, p. 262]. If the original value group is archimedean, the value group of this overfield will be archimedean.

\section{BIBLIOGRAPHY}

1. I. Kaplansky, Maximal fields with valuations, Duke Math. J. vol. 9 (1942) pp. 303-321.

2. - Maximal fields with valuations, II, Duke Math. J. vol. 12 (1945) pp. 243-248.

3. P. W. Carruth, Arithmetic of ordinals with applications to the theory of ordered abelian groups, Bull. Amer. Math. Soc. vol. 48 (1942) pp. 262-271.

4. H. Hahn, Über die Nichtarchimedischen Grössensysteme, Sitzungsberichte der Mathematischen Naturwissenschaftliche Klasse der Kaiserlichen Akademie der Wissenschaften, Vienna vol. 116 (II A) (1907) pp. 601-655.

5. W. Krull, Allgemeine Bewertungstheorie, J. Reine Angew. Math. vol. 167 (1932) pp. 160196.

6. S. MacLane, Subfields and automorphism groups of p-adic fields, Ann. of Math. vol. 40 (1939) pp. 423-442.

7. O. Teichmüller, Diskret Bewertete Perfekte Körper mit Unvollkommenen Restklassenkörper, J. Reine Angew. Math. vol. 176 (1937) pp. 141-152.

8. B. L. van der Waerden, Moderne Algebra, vol. 1, New York, 1943.

UNIVERSITY OF ILLINOIS,

URBANA, ILL. 